Research Article

\title{
Utility of modified mid-pregnancy body mass index in predicting obstetric outcomes in Middle Eastern population
}

\begin{abstract}
Objective: To test the value of mid-pregnancy maternal body mass index (BMI) and weight gain in the second half of pregnancy in predicting pregnancy outcomes among Middle Eastern population where pre-gravid BMI was not available.

Methods: Retrospective analysis of the obstetric outcomes of 290 women with singleton pregnancies who were categorized into underweight (UW), normal weight (NW), overweight $(\mathrm{OW})$ and obese $(\mathrm{OB})$ according to the BMI at 24-28 weeks of gestation and compared with recommended pre-gravid values by Institute of Medicine (2009).

Results: The results showed that the gestational diabetes rate was significantly lower among the UW $(2.4 \%)$ compared with the NW $(8.8 \%, p<0.05)$, the OW $(14.3 \%, p=0.006)$, and OB $(20.5 \%, 0.001)$. The risk of having macrosomia was eight times higher among OW compared with NW women. The C-section rate was lowest among the UW group but there was no significant difference in terms $\mathrm{C}$-section and low birth weight between groups. The obstetric outcomes among women whose weight gain was above the 90th and below the tenth centiles were not different.
\end{abstract}

Conclusion: The prediction of obstetric outcomes by mid-pregnancy BMI is comparable to pre-gravid BMI. The results are encouraging, and further larger studies are needed to confirm the applicability.

Keywords: mid-pregnancy body mass index, obstetric outcomes, maternal weight gain
Volume 7 Issue I - 202I

\author{
Muzibunnisa A Begam,' Sultan M \\ Salahudheen ${ }^{2}$ \\ 'Department of Obstetrics\& Gynecology, Mediclinic Airport \\ road Hospital, United Arab Emirates \\ ${ }^{2}$ Department of Pharmacy, Sheikh Zayed Hospital, United Arab \\ Emirates
}

Correspondence: Muzibunnisa A Begam, MD, FRCOG, CESR MFM UK, Consultant, Fetal Medicine Unit, Department of Obstetrics\& Gynecology, Mediclinic Airport road Hospital,Abu Dhabi, United Arab Emirates, Email drmuzi@yahoo.com

Received: July 06, 2020 | Published: February 24, 202
Synopsis: Predictive value of obstetric outcomes by second trimester body mass index is similar to pre-gravid BMI.

Abbreviations: NW, normal weight; OW, overweight; OB, obese; UW, underweight; BMI, body mass index.

\section{Introduction}

Maternal pre-gravid nutrition and gestational weight gain have been reported to have significant impact on pregnancy outcomes. The Institute of Medicine (IOM) ${ }^{1}$ categorized women on the basis of their pre-gravid body mass index (BMI) and provided guidelines for optimal gestational weight gain (Table 1). Several studies have reported on the validity and applicability of these recommendations in predicting the outcomes of pregnancies. ${ }^{2,3}$ However, these recommendations are only applicable where the pre-gravid BMI is known and they are not applicable where the patient attends for the first time when the pregnancy is already advanced. The use of recalled pre-gravid in place of measured pre-gravid weight has produced different results. ${ }^{4}$ In our obstetric practice, as in other developing countries, many of the expectant mothers present for first prenatal visit in the second or third trimester when they feel assured of fetal viability. Furthermore, many of the expectant mothers have neither had a pre-gravid check nor can recall their pre-gravid body weight and for these reasons, the IOM recommendations are not directly applicable. The clinical usefulness of routine weighing of patients in the antenatal care remains controversial. While some studies ${ }^{5,6}$ found a correlation between changes in maternal weight during pregnancy and pregnancy outcomes, others ${ }^{7}$ found no such relationship and regard weight measurements as a source of unnecessary anxiety. It has also been suggested that because maternal anthropometry differs by ethnic groups, different recommendations should be made for each ethnic population. ${ }^{8}$ Our obstetric populations are derived mainly from the Arabic and Asian stock, while the IOM recommendations are based on a predominantly Caucasian population.

Table I Recommended total weight gain ranges for pregnant women according to prepregnancy body mass index (BMI) (IOM 2009)

\begin{tabular}{lll}
\hline & \multicolumn{2}{c}{ Recommended weight gain } \\
\hline Prepregnancy BMI $\left(\mathbf{k g} / \mathbf{m}^{2}\right)$ & $\mathbf{K g}$ & $\mathbf{l b}$ \\
\hline$<18.5$, Underweight & $12.5-18.0$ & $28-40$ \\
18.5-24.9, normal weight & $11.5-16.0$ & $25-35$ \\
$25-29.9$, overweight & $7.0-11.5$ & $15-25$ \\
$\geq 30.0$ obese & $5-9$ & ||$-20$ \\
\hline
\end{tabular}

The purpose of this study was to explore the relationship between mid-pregnancy maternal BMI, changes in maternal weight gain in the second half of pregnancy and pregnancy outcomes among predominantly Arab and Asian population in order to test the hypothesis that the more readily available mid-pregnancy BMI is as predictive of pregnancy outcomes as the pre-gravid value.

\section{Materials and methods}

This was a retrospective cross-sectional study of deliveries conducted at our Hospital during the period $1^{\text {st }}$ January to $31^{\text {st }}$ December 2017. Routine prenatal clinical evaluation of women at first visit includes detailed history, height and weight measurement, complete physical examination, urine dipstick check, basic pregnancy 
laboratory workup and initial obstetric ultrasound assessment of the fetus to confirm gestational age. Universal screening for gestational diabetes mellitus (GDM) with 75 gms. Oral glucose tolerance test (OGTT) is offered between 24-28 weeks gestational age. Maternal weight is obtained in normal clothing and recorded at each clinic visit. Detailed delivery data including course of pregnancy, gestational age, onset of labor, duration and method of delivery, newborn gender and birth-weight and complications are logged in the labor ward register.

Four hundred chart numbers of women who delivered during this twelve month period were randomly selected by choosing every fifth number in the record book. Using the inclusion criteria of women who had singleton pregnancies, initial visit before 24-28 weeks of gestation, at least four regular further follow-ups and delivery in the hospital, two hundred and ninety (290) were selected. Those who did not meet these criteria were de-selected. The information obtained from the charts included maternal age, parity, significant medical or obstetrical complications, body weight between 24-28 weeks of gestation and 6 weeks later. The obstetric outcomes analyzed were primary caesarean delivery, glucose intolerance (IGTT), GDM, hypertension, low birth weight (LBW) and macrosomia.

The IOM guidelines provide categories of women as underweight, normal, overweight and obese according to the pre-gravid BMI. The guidelines also recommend the ideal weight gain per week during pregnancy for each category. This information was used to derive the expected BMI of a mother in each of the categories at 24-28 weeks gestation (Table 2).

Table 2 Distribution according to BMI at 24-28 weeks gestation- age and parity

\begin{tabular}{llll}
\hline & No & Age (yrs + SD & Parity \\
\hline Underweight (BMI <25) & 85 & $24.5+4.6$ & $1.3+1.7$ \\
Normal weight (BMI 25-29.9) & 103 & $26.0+5.0$ & $2.0+2.3$ \\
Overweight (BMI 30-35) & 63 & $28.2+6.0$ & $2.9+2.6$ \\
Obese (BMI>35) & 39 & $29.7+7.0$ & $3.6+3.1$ \\
TOTAL & 290 & $26.6+5.7$ & $2.2+2.5$ \\
\hline
\end{tabular}

The derived BMI was used, in place of the pre-gravid weights, to categorize the patients into, underweight (UW), normal weight (NW), overweight (OW) and obese (OB). All data were logged using the SPSS Statistical package. Pregnancy outcomes of women in the different categories were compared (Tables $3 \& 4)$. The change in BMI per month was calculated for each patient using the difference between the weight at 24-28 weeks gestation and six weeks later. The differences were ranked into centiles. Pregnancy outcomes of patients with weight changes above $90^{\text {th }}$ centile and those below $10^{\text {th }}$ centile were respectively compared with those of patients in the $10^{\text {th }}$ to the $90^{\text {th }}$ centile range, (Table 5). Differences between groups were tested using the Student's t test and between proportions, using the Chi Square test. Yates correction was applied if the numbers were small.

\section{Results}

The medical records of 290 out of the 400 women provided information that met the inclusion criteria and these are the subjects of this study. Table 2 shows the clinical data among all the subjects at 24-28 weeks gestation. Although the parity, age appear to increase progressively, in the order $\mathrm{UW}<\mathrm{NW}<\mathrm{OW}<\mathrm{OB}$, the differences were not clinically significant. Table 3 shows the caesarean section rates and newborn weights whereas Table 4 compares the Glucose intolerance rates among these women. The low birth-weight (defined as birth weight less than 2500 gms. at completed 37 weeks gestation) rate was also not significantly different among the groups. There was no statistical difference in the rates of macrosomia (birth-weight greater than $4000 \mathrm{gm}$ ) among the women in the groups of UW, NW and OB. However, the women in OW group had significantly higher rates of macrosomia than the UW (14.3\% vs $1.2 \%, \mathrm{p}-0.002)$ and NW $(14.3 \%$ Vs $1.9 \%, \mathrm{p}=0.003)$.

The caesarean section rate was lowest among the UW group, but the differences between groups were not statistically different. The gestational diabetes (GDM) rate was significantly lower among the UW $(2.4 \%)$ compared with the NW $(8.8 \%, \mathrm{p}<0.05)$, the OW $(14.3 \%, p=0.006)$, and OB $(20.5 \%, 0.001)$. The GDM rate was also significantly $(\mathrm{p}=0.035)$ among the NW $(8.8 \%)$ compared with the OB $(20.5 \%)$, but not from the OW $(14.3 \%, p=0.1)$. There was no significant statistical difference either in the rates of impaired glucose tolerance or in the rate of need for insulin to control diabetes among the groups. Twenty-eight (28) mothers were in the $10^{\text {th }}$ centile and below and 44 were in the $90^{\text {th }}$ centile and above of weight gain in the last half of pregnancy. There was no significant statistical difference in the rates of low birth-eight, caesarean section rate, abnormal glucose patterns, high blood pressure and macrosomia when these two groups were compared among each other and when compared with mothers in the $10^{\text {th }}$ to $90^{\text {th }}$ centile range.

Table 3 Distribution according to BMI at 24-28 weeks gestation- caesarean section and newborn weights

\begin{tabular}{llllll}
\hline & No & C/S \% & BW $(\mathrm{g})+$ SD & Macrosomia* \% & LBW \% \\
\hline Underweight (BMI <25) & 85 & 9.4 & $3045+472$ & 1.2 & 4.7 \\
Normal weight (BMI 25-29.9) & 103 & 11.7 & $3172+445$ & 1.9 & 2.9 \\
Overweight (BMI 30-35) & 63 & 15.9 & $3398+598$ & 14.3 & 0 \\
Obese (BMI>35) & 39 & 12.8 & $3275+443$ & 2.6 & 2.6 \\
TOTAL & 290 & 12.1 & $3197+505$ & 4.5 & 2.8 \\
\hline
\end{tabular}

Underweight Vs Overweight $p=0.002$, Vs Obese $p=0.04$ : Normal weight Vs Overweight, $p=0.003$

Table 4 Distribution according to BMI at 24-28 weeks gestation- abnormal blood sugar rates

\begin{tabular}{lllll}
\hline & No & IGTT \% & GDM* \% & IDDM \% \\
\hline Underweight $(\mathrm{BMI}<25)$ & 85 & 3.6 & 2.4 & 0 \\
Normal weight $($ BMI 25-29.9) & 103 & $\mathrm{I}$ & 8.8 & $\mathrm{I}$ \\
\hline
\end{tabular}


Table continued...

\begin{tabular}{lllll}
\hline & No & IGTT \% & GDM* \% & IDDM \% \\
\hline Overweight (BMI 30-35) & 63 & 3.2 & 14.3 & 3.2 \\
Obese (BMI>35) & 39 & 1 & 20.5 & 1 \\
ALL & 290 & 2.1 & 9.6 & 1.3 \\
\hline
\end{tabular}

*Under weight $V_{s}$ Normal Weight $(P<0.05), V s$ Overweight $p=0.006$, Vs Obese $p=0.001$ : Normal weight Vs Obese $p=0.35$

Table 5 Changes in BMI per month and pregnancy outcomes

\begin{tabular}{|c|c|c|c|c|c|c|c|c|c|c|c|}
\hline BMI change/month & $\mathbf{N}$ & Age & Parity & LBW & C/S \% & IGTT \% & GDM \% & IDDM \% & HBP \% & BW(g) & Macrosomia \% \\
\hline 90th centile and above & 30 & $23.9+4.7$ & $1.4+2.3$ & $3.3(I)$ & 6.7 & 3.3 & 0 & 0 & 6.7 & $3180+510$ & 0 \\
\hline I0th centile and below & 44 & $26.0+4.9$ & $2.1+2.6$ & 0 & 6.8 & 4.5 & 9.1 & 0 & 4.5 & $3212+453$ & 2.3 \\
\hline
\end{tabular}

\section{Discussion}

Pre-gravid BMI and gestational weight gain impact on pregnancy and perinatal outcomes. ${ }^{2,3,9,10}$ Ogunyemi et al., ${ }^{2}$ reported that pregravid obesity (BMI $\geq 30$ ) was associated with hypertensive disorders, GDM, caesarean delivery and high mean birth weight while low pregravid $\mathrm{BMI}(\mathrm{BMI} \leq 20)$ was associated with low birth weight (LBW) in the rural black population. Similar observations were made among the Japanese population. ${ }^{11}$ Pre-gravid BMI has been shown to be associated with increased gestational hypertension, birth weight and neonatal fat mass, independent of OGTT values. ${ }^{12}$

Since many of the subjects presented for the first time in pregnancy, these authors have used recalled pre-gravid body weight in calculating the pre-gravid BMI. Although earlier studies reported that this method was valid, ${ }^{13,14}$ further data indicate that the use of measured pre-pregnancy weight on newborn size produces different results than does the use of recalled pre-pregnancy weight. ${ }^{4}$ Because the pre-gravid BMI is usually unavailable in most of our obstetric population, we have used the extrapolated ideal BMI at 24-28 weeks of gestation based on IOM recommendations on weight gain as surrogate reference for BMI. At 24-28 weeks gestation, women with BMI 30-35 (overweight) and $\mathrm{BMI} \geq 35$ (obese) were at higher risk of GDM and caesarean delivery. The risk of having macrosomic fetus was eight times higher among overweight women compared with normal weight women. These results are similar to previous observations using the pre-gravid BMI and perinatal outcomes ${ }^{2,3,8}$ and the results suggest that the mid-trimester BMI may be as predictive of these adverse pregnancy outcomes as pre-gravid BMI.

While the association between pre-gravid BMI and perinatal outcomes is well accepted, controversies still exist ${ }^{7}$ regarding gestational weight gain and its impact on pregnancy outcomes. Some authors have suggested that weight should be monitored during pregnancy regardless of the pre-pregnancy BMI. ${ }^{8}$ On the other hand, Mukarami et al., ${ }^{11}$ reported that weight gain during pregnancy did not show any significant influence on the perinatal outcomes and Dawes et $\mathrm{al}^{7}{ }^{7}$ have recommended that the maternal weight need be recorded only at booking and subsequent routine weighing of patients only serves to raise anxiety and should cease. The current study shows that no statistical differences in the rates of low birth-weight rate, GDM, glucose intolerance and macrosomia among women whose weight gain was above the $90^{\text {th }}$ centile and those whose weight gain per month was below the tenth centile of in the $10^{\text {th }}$ to $90^{\text {th }}$ centile range. From the small sample size, it seems that weight gain during pregnancy was not predictive of these adverse pregnancy outcomes and measurement either pre-gravid or mid-pregnancy BMI predicts the adverse perinatal outcomes better than weight gain during pregnancy. This supports the position that maternal weight need be recorded only at booking. ${ }^{7}$

\section{Conclusion}

These data suggest that the second trimester BMI has similar predictive value as pre-gravid BMI for obstetric outcomes and be utilized for this purpose in planning the care of pregnant women where pre-gravid BMI is not available. The results are encouraging and further larger studies are needed to confirm the applicability.

\section{Acknowledgements}

The staffs of the medical records and the statistical departments are acknowledged for their immense help and support.

\section{Conflicts of interest}

Author declares that there is no conflict of interest.

\section{Funding}

None.

\section{References}

1. IOM. Weight Gain During Pregnancy: Reexamining the Guidelines. Institute of Medicine (US) and National Research Council (US) and Committee to Reexamine IOM Pregnancy Weight Guidelines, Washington, D.C.; 2009

2. Ogunyemi D, Hullett S, Leeper J, et al. Pre-pregnancy body mass index, weight gain during pregnancy, and perinatal outcome in a rural black population. J Matern Fetal Med. 1998;7(4):190-193.

3. Margaretha Haugen, Anne Lise Brantsæter, Anna Winkvist, et al Associations of pre-pregnancy body mass index and gestational weight gain with pregnancy outcome and postpartum weight retention: a prospective observational cohort study. BMC Pregnancy Childbirth. 2014;11(14):201.

4. Brown JE, Murtaugh MA, Jacobs D JR, et al. Variation in newborn size according to pregnancy weight change by trimester. Am J Clin Nutr. 2002;76(1):205-209.

5. Ehrenberg HM, Dierker L, Milluzzi C, et al. Low maternal weight, failure to thrive in pregnancy, and adverse pregnancy outcomes. $A m J$ Obstet Gynecol. $2003 ; 189: 1726-1730$.

6. Yang W, Han F, Gao X, et al. Relationship Between Gestational Weight Gain and Pregnancy Complications or Delivery Outcome. Sci Rep. 2017;7(1):12531.

7. Dawes MG, Grudzinskas JG. Repeated measurement of maternal weight during pregnancy. Is this a useful practice? Br J Obstet Gynecol. 1991;98:189-194.

8. Yekta Z, Ayatollahi H, Porali R, et al. The effect of pre-pregnancy body mass index and gestational weight gain on pregnancy outcomes in urban care settings in Urmia-Iran. BMC Pregnancy Childbirth. 2006;6:15. 
9. Farah N, Maher N, Barry S, et al. Maternal morbid obesity and obstetric outcomes. Obes Facts. 2009;2(6):352-354.

10. Li N, Liu E, Guo J, et al. Maternal prepregnancy body mass index and gestational weight gain on pregnancy outcomes. PLoS One. 2013;20;8(12):e82310.

11. Mukarami M, Ohmichi M, Takahashi T, et al. Pre-pregnancy body mass index as an important predictor of perinatal outcomes in Japanese women. Arch Gynecol Obstet. 2005;271:311-315.
12. Stuebe AM, Landon MB, Lai Y, et al. Maternal BMI, glucose tolerance, and adverse pregnancy outcomes. Am J Obstet Gynecol. 2012;207(1):62. e1-7.

13. Stunkard A, Albaum J. The accuracy of self reported weights. Am J Clin Nutr. 1981;31:1593-1599.

14. Wing R, Epsein LH, Ossip DJ, et al. Reliability and validity of self report and observers estimates of relative weight. Addict Behav. 1979;4:133140 